See discussions, stats, and author profiles for this publication at: https://www.researchgate.net/publication/304399044

\title{
The Monoglyceride Content Affects the Self-Assembly Behavior, Rheological Properties, Syringeability, and Mucoadhesion of In Situ-Gelling Liquid Crystalline Phase
}

Article in Journal of Pharmaceutical Sciences · June 2016

DOI: 10.1016/j.xphs.2016.05.005

\section{CITATIONS}

4

7 authors, including:

Kariane Nunes

Universidade Federal do Oeste do Pará

4 PUBLICATIONS 9 CITATIONS

SEE PROFILE

victor hugo vitorino Sarmento

Universidade Federal de Sergipe

53 PUBLICATIONS 872 CITATIONS

SEE PROFILE
READS

159

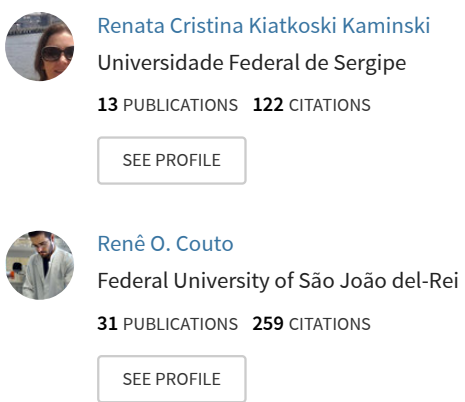

Some of the authors of this publication are also working on these related projects:

Síntese e caraterização de híbridos polímero argila e sua influência na germinação de sementes View project

Textura e Estabilidade Térmica de Nanopartículas de $\mathrm{SnO} 2$ Modificadas por Tiron ${ }^{\circledast}$ View project 
Pharmaceutics, Drug Delivery and Pharmaceutical Technology

\title{
The Monoglyceride Content Affects the Self-Assembly Behavior, Rheological Properties, Syringeability, and Mucoadhesion of In Situ-Gelling Liquid Crystalline Phase
}

\author{
Kariane M. Nunes ${ }^{1,{ }^{*}}$, Cristian C.C. Teixeira ${ }^{1}$, Renata C.K. Kaminski ${ }^{2}$, \\ Victor H.V. Sarmento ${ }^{2}$, Renê O. Couto ${ }^{3}$, Sandra H. Pulcinelli ${ }^{4}$, Osvaldo Freitas ${ }^{1}$ \\ ${ }^{1}$ Department of Pharmaceutical Sciences of Ribeirão Preto, USP-University of São Paulo, Ribeirão Preto, São Paulo, Brazil \\ ${ }^{2}$ Department of Chemistry, UFS - Federal University of Sergipe, Itabaiana, Sergipe, Brazil \\ ${ }^{3}$ Department of Pharmaceutical Sciences, Health Science Center, UEL-State University of Londrina, Londrina, Paraná, Brazil \\ ${ }^{4}$ Department of Pharmaceutical Sciences, Chemistry Institute of Araraquara, UNESP-São Paulo State University, Araraquara, São Paulo, Brazil
}

\section{A R T I C L E I N F O}

\section{Article history:}

Received 16 February 2016

Accepted 3 May 2016

Available online 21 June 2016

\section{Keywords:}

responsive delivery systems

mucosal drug delivery

controlled release

lipids

surfactants

factorial design

injectables

hydration

physical characterization

\begin{abstract}
A B S T R A C T
This article reports the development of a precursor liquid crystalline system based on a mixture of monoglycerides (MO) and Cremophor ${ }^{\circledR}$ (CREM) that exhibits in situ gelation to a liquid crystalline phase. The effects of different MO/CREM ratios and the water content $\left(W_{C}\right)$ on several performance characteristics were investigated with a full factorial design. The formulations were characterized by polarized light microscopy, small-angle X-ray scattering, and water uptake assays. Rheological, syringeability, and mucoadhesion evaluation were also performed. The polarized light microscopy and small-angle X-ray scattering results for average and high MO/CREM ratios (2.1 and 4.0, respectively) indicated the coexistence of phases in transition to the liquid crystalline phase, independently of the $\mathrm{W}_{\mathrm{c}}$. These systems became more viscous after taking up water, showing peaks characteristic of a cubic phase. Systems that had average and high MO/CREM ratios also exhibited shear-thinning behavior and high elasticity. Most systems showed suitable mucoadhesion for buccal purposes. Response surface methodology results demonstrated that the relative contribution of MO was the principal factor that affected the performance of the system. Accordingly, these precursor systems with average to high MO/CREM ratios and an average $\mathrm{W}_{\mathrm{C}}(10 \% \mathrm{w} / \mathrm{w})$ demonstrated physicochemical and mucoadhesive properties that could enable them to be used as an in situ-gelling controlled drug delivery platform.
\end{abstract}

๑ 2016 American Pharmacists Association ${ }^{\circledR}$. Published by Elsevier Inc. All rights reserved.

\section{Introduction}

In situ gelling systems are an attractive approach for the design of formulations for sustained drug delivery via different administration routes. ${ }^{1-4}$ The viscosity of this type of system can be modulated by the amount of water it contains, ${ }^{5}$ by temperature, and by $\mathrm{pH}$ changes ${ }^{6}$ and can undergo a transition to gel on contact with the bodily fluid at a mucosal site. ${ }^{1}$ The administration of the drug delivery system (DDS) into confined spaces, for example, within the vaginal ${ }^{7,8}$ and nasal ${ }^{1,9}$ cavities, periodontal pocket, $^{10,11}$ or conjunctival sac, ${ }^{2}$ is a complex task and remains as a challenge for formulation scientists.

\footnotetext{
This article contains supplementary material available from the authors by request or via the Internet at http://dx.doi.org/10.1016/j.xphs.2016.05.005.

* Correspondence to: K.M. Nunes (Telephone: +55-93 2101 4947).

E-mail address: kariane.nunes@gmail.com (K.M. Nunes).
}

Several limitations due to anatomy and physiology must be overcome during studies of formulations, including the limited size and depth of the area and the flow of the relevant bodily fluid. ${ }^{12}$ Thus, such systems must combine 2 main characteristics: (1) fluidity, enabling ease of application into the desired site via a syringe and (2) suitable mucoadhesion, to persist for an adequate time at the site.

In this regard, hydrophilic polymers, such as Carbopol ${ }^{\circledR}$, sodium alginate, Poloxamer ${ }^{\circledR}$, hydroxypropylmethylcellulose, and hydroxyethylcellulose, have been widely studied. ${ }^{13,14}$ However, the high hydrophilicity and hydration capacity of these polymers facilitate the erosion of the system in aqueous medium, resulting in rapid drug release. ${ }^{15}$ It can also induce irritation in the administration region and patient discomfort, which may impair the efficacy of the clinical treatment.

To address these concerns, other polymers and copolymers have been studied, such as polyesters and their copolymers with 
poly(ethylene) (PEG), ${ }^{16}$ poly(lactic acid),${ }^{17}$ and poly[(lactic acid)-co(glycolic acid)], ${ }^{18}$ as well as pseudo poly(amino acids) ${ }^{19}$ and amphiphilic lipids, for example, monoglycerides (MO). ${ }^{20}$

MO are polar, amphiphilic lipids which have remarkable chemical and physical stability, nontoxicity, biocompatibility, biodegradability, and the extraordinary ability to self-assemble into a viscous liquid crystalline phase (LCP) in the presence of excess water $^{21,22}$ forming a fluid precursor system (FPS). Their use in a gelling system for the development of new formulations has intensified over the last few years in both academic research and industry, mainly for pharmaceutical application. ${ }^{23-27}$

Depending on the amount of water in the mesophase, the properties of the solid and liquid states are combined in different ways to create a phase with 1 of 3 structures: lamellar, hexagonal, or cubic. ${ }^{28-30}$ This drug delivery technology can control the speed and extension of drug release at the administration site and provide stability against disintegration in the biological environment. ${ }^{31,32}$

Although FPS based on MO enable drugs of different polarities and molecular weight to be carry within their compartments, ${ }^{33}$ the release efficiency of poorly water-soluble drugs is low. This limitation can be avoided by the addition of a surfactant ${ }^{34,35}$ such as Cremophor ${ }^{\circledR}$ EL (CREM), which is a nonionic surfactant widely used as a pharmaceutical excipient in oral, parenteral, and topical formulations because of its high solubility and recognized nontoxicity and nonirritability. ${ }^{36}$

To study the applicability of these systems for mucosal controlled drug delivery via a syringe, we developed a FPS from MO, CREM, and water. The effects of the MO/CREM ratio ( $w / w)$ and the water content $\left[\mathrm{W}_{\mathrm{C}}(\% \mathrm{w} / \mathrm{w})\right]$ on the self-assembly profile, rheological properties, syringeability, hydration, and mucoadhesion of various formulations were systematically evaluated using a chemometric approach.

\section{Experimental}

\section{Chemicals}

MO (Myverol ${ }^{\circledR}$ 18-92 K), were supplied by Kerry do Brasil (TrêsCorações, Brazil). Myverol ${ }^{\circledR} 18-92 \mathrm{~K}$ has a purity of $93 \% \mathrm{MO}$ [containing 65\% glyceryl monolinoleate, 3\% glycerylmonooleate, 6\% monoglyceride (C16), 4\% monoglyceride (C18), 1\% monoglyceride (C20), and 1\% monoglyceride (C18:3)], 6\% diglycerides and triglycerides that have the same fatty acid profile as the MO and other minor $(<1 \%)$ free fatty acids, and glycerol. Cremophor ${ }^{\mathbb{R}}$ EL $($ CREM) was purchased from Sigma-Aldrich (Steinheim, Germany).

\section{Experimental Design, Preparation of FPS, and Response Surface Methodology}

The experimental design was a 2 factor and 3-level full factorial design with 2 replicates of the central point $\left(3^{2}+2\right)$. The factors studied (independent variables) and their levels were as follows: $X_{1}$, the MO/CREM ratio at $0.25,2.1$, and $4(\mathrm{w} / \mathrm{w})$ and $X_{2}$, the $\mathrm{W}_{\mathrm{C}}$ at $5 \%$, $10 \%$, and $15 \%(\mathrm{w} / \mathrm{w})$. The full factorial design matrices with coded and noncoded values of each factor studied are summarized in Table 1 . The trials were randomized to minimize the effects of unexplained variability due to extraneous factors on the observed responses.

The FPSs were prepared from appropriate amounts of MO melted at $45 \pm 1.0^{\circ} \mathrm{C}$ in a water bath with CREM and warm water. The components were completely homogenized and kept at room temperature $\left(25 \pm 1.0^{\circ} \mathrm{C}\right)$ for $24 \mathrm{~h}$ for further evaluation. Eleven FPS (Table 1) were prepared and labeled using the notation MCxWy. $\mathrm{MCX}$ indicated the MO/CREM ratio $(0.25,2.1$, and 4.0$)$, and $\mathrm{Wy}$ indicated the $\mathrm{W}_{\mathrm{C}}(5 \%, 10 \%$, and $15 \%, \mathrm{w} / \mathrm{w})$. For example, MC025W15 means that the FPS was prepared with MO/CREM ratio of $0.25 \%$ and $15 \% W_{C}$. Viscous LCP were obtained from $500 \mathrm{mg}$ of preprepared FPS in contact with excess water $(40 \% \mathrm{w} / \mathrm{w})$.

Table 1 summarizes the actors coded for the ANOVA by the response surface methodology (RSM) following the coding rule given by Equation 1:

Codedvalue $=\frac{(\text { uncodevalue }-0.5 \times(\text { highvalue }+ \text { lowvalue })}{0.5 \times(\text { highvalue }- \text { lowvalue })}$

An ANOVA/RSM on the experimental data was performed using the visual general linear model from the Statistica 12 (StatSoft Inc., Tulsa, OK) software package. The mathematical models for each response were evaluated using a multiple regression method. The response function applied was a linear polynomial equation, given by Equation 2:

$Y=\beta_{0}+\sum_{i=1}^{k} \beta_{i} x_{i}+\sum \beta_{i j} x_{i} x_{j}$

$Y$ is the dependent variable; $\beta_{0}$ is the constant term, $k$ is the number of variables, $\beta_{\mathrm{i}}$ represents the coefficients of the linear parameters,

Table 1

Full Factorial Design Matrices and Results of Liquid Crystalline Phase Precursor's Performance Indicators ${ }^{\mathrm{a}}$

\begin{tabular}{|c|c|c|c|c|c|c|c|c|c|c|}
\hline Run Order $^{\mathrm{b}}$ & $\begin{array}{r}\text { Standard } \\
\text { Order }^{c}\end{array}$ & FPS Code & $X_{1} \cdot \mathrm{MO} / \mathrm{CREM}(-)$ & $X_{2} \cdot \mathrm{W}_{\mathrm{C}}[\%(\mathrm{w} / \mathrm{w})]$ & $k($ Pa.s) & $n(-)$ & $\mathrm{W}_{\mathrm{S}}(\mathrm{N} . \mathrm{mm})$ & Wu 24 h $(\%$ w/w $)$ & $\mathrm{S}_{\mathrm{M}}(\mathrm{N})$ & $\mathrm{W}_{\mathrm{M}}(\mathrm{N} . \mathrm{mm})$ \\
\hline 8 & 1 & MC025W5 & $0.25(-1)$ & $5(-1)$ & $0.72 \pm 0.02$ & $0.98 \pm 0.004$ & $152.2 \pm 5.1$ & Undetermined & $0.14 \pm 0.03$ & $0.59 \pm 0.17$ \\
\hline 5 & 2 & MC025W10 & $0.25(-1)$ & $10(0)$ & $0.69 \pm 0.02$ & $0.99 \pm 0.001$ & $187.8 \pm 3.1$ & Undetermined & $0.09 \pm 0.01$ & $0.36 \pm 0.04$ \\
\hline 9 & 3 & MC025W15 & $0.25(-1)$ & $15(+1)$ & $0.96 \pm 0.04$ & $0.97 \pm 0.002$ & $187.3 \pm 8.1$ & Undetermined & $0.13 \pm 0.03$ & $0.67 \pm 0.15$ \\
\hline 2 & 4 & MC21W5 & $2.1(0)$ & $5(-1)$ & $2.28 \pm 0.23$ & $0.75 \pm 0.01$ & $103.5 \pm 5.9$ & $8.9 \pm 1.8$ & $0.17 \pm 0.05$ & $1.0 \pm 0.36$ \\
\hline 4 & 5 & MC21W10 & $2.1(0)$ & $10(0)$ & $1.74 \pm 0.10$ & $0.77 \pm 0.003$ & $111.5 \pm 2.7$ & $30.4 \pm 1.6$ & $0.18 \pm 0.01$ & $0.91 \pm 0.26$ \\
\hline 7 & 6 & MC21W15 & $2.1(0)$ & $15(+1)$ & $1.58 \pm 0.01$ & $0.84 \pm 0.01$ & $156.1 \pm 4.1$ & $9.8 \pm 4.0$ & $0.16 \pm 0.03$ & $0.92 \pm 0.28$ \\
\hline 1 & 7 & MC40W5 & $4.0(+1)$ & $5(-1)$ & $13.51 \pm 1.35$ & $0.51 \pm 0.02$ & $182.8 \pm 6.2$ & $15.0 \pm 4.4$ & $0.17 \pm 0.01$ & $0.83 \pm 0.05$ \\
\hline 3 & 8 & MC40W10 & $4.0(+1)$ & $10(0)$ & $15.08 \pm 2.51$ & $0.52 \pm 0.01$ & $193.0 \pm 3.5$ & $29.5 \pm 1.4$ & $0.19 \pm 0.04$ & $1.17 \pm 0.17$ \\
\hline 10 & 9 & MC40W15 & $4.0(+1)$ & $15(+1)$ & $530.5 \pm 73.26$ & $2.10^{-8} \pm 1.10^{-8}$ & $484.3 \pm 18.4$ & $27.3 \pm 1.8$ & $0.15 \pm 0.01$ & $0.94 \pm 0.17$ \\
\hline 11 & 10 & MC21W10 & $2.1(0)$ & $10(0)$ & $1.83 \pm 0.12$ & $0.78 \pm 0.001$ & $103.1 \pm 5.9$ & $33.9 \pm 5.4$ & $0.17 \pm 0.02$ & $0.97 \pm 0.22$ \\
\hline 6 & 11 & MC21W10 & $2.1(0)$ & $10(0)$ & $1.63 \pm 0.05$ & $0.77 \pm 0.004$ & $109.9 \pm 5.3$ & $29.3 \pm 1.1$ & $0.18 \pm 0.01$ & $0.93 \pm 0.09$ \\
\hline
\end{tabular}

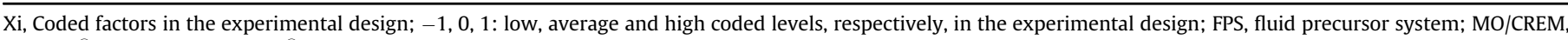

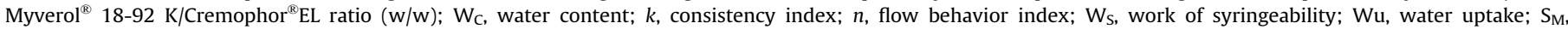
mucoadhesion strength; $W_{M}$, mucoadhesion work.

a Results are presented as average \pm standard deviation.

b Randomized.

c Nonrandomized. 
$\beta_{\mathrm{ii}}$ represents the coefficients of the quadratic terms, and $\beta_{\mathrm{ij}}$ represents the coefficients of the interaction parameters.

The significance of the equation parameters for each response variable was analyzed by an F-test. Only the factors with a significance higher than or equal to $5 \%(p \leq 0.05)$ were considered. The adequacy of the model was checked to account for the coefficient of determination $\left(\mathrm{R}^{2}\right)$.

\section{Polarized Light Microscopy}

The FPS and viscous LCP were placed on a glass microscope slide, carefully covered with a coverslip to distribute the contents by application of pressure and analyzed by polarized light microscopy (PLM) at room temperature using a Carl Zeiss Axioplan 2 microscope (Oberkochen, Germany) equipped with a digital camera.

\section{Small-Angle X-Ray Scattering}

Measurements were performed at room temperature in a small-angle X-ray scattering (SAXS1) beamline located at the National Laboratory of Synchrotron Light (LNLS, Campinas, Brazil). The LNLS beamline was equipped with a monochromator ( $\lambda=1.5498 \AA$ ), a vertical detector located approximately $580 \mathrm{~mm}$ from the sample and a multichannel analyzer that registered the scattering intensity $I(q)$ as a function of the scattering vector, $q$.

The samples were injected into the sample holder with mica windows placed perpendicular to the X-ray beam. The data were normalized for beam decay, detector sensitivity, and sample transmission. Silver behenate powder was used as a standard to calibrate the sample-to-detector distance, the detector tilt, and the incident beam position. The parasitic scattering intensity, resulting from the cell windows and air, was subtracted from the total scattering intensity.

\section{Rheological Measurements}

Steady shear rate and dynamic frequency sweep tests were performed using a stress controlled rheometer AR2000ex (TA Instruments, Surrey, England) in the flow and oscillatory modes, respectively, with a parallel steel plate geometry $(40 \mathrm{~mm})$ and a gap of $500 \mu \mathrm{m}$ between the plates. FPS samples were carefully applied to the lower plate of the rheometer and analyzed at room temperature, ensuring the minimum shear and a 5-min rest time before each measurement was allowed.

Steady shear rate sweep tests (upward and downward) were carried out over a range of shear rates $\left(0-2000 \mathrm{~s}^{-1}\right)$. The shear rate was increased over a period of $150 \mathrm{~s}$ and held at the upper limit for $20 \mathrm{~s}$, then decreased for a period of $150 \mathrm{~s}$. The flow properties were determined for each measurement at least in triplicate. The experimental upward flow curves for the samples are described by a power law (Oswald-de-Waele equation), as follows:

$\sigma=k \cdot \dot{\gamma}^{n}$

$\sigma$ is the shear stress (Pa), $k$ is the consistency index (Pa.s), $\dot{\gamma}$ is the shear rate $\left(\mathrm{s}^{-1}\right)$, and $n$ is the flow behavior index (dimensionless). ${ }^{37}$

In the dynamic frequency sweep tests, the linear viscoelastic region was identified via an oscillatory stress sweep at a fixed frequency $(1 \mathrm{~Hz})$. Then, the samples were subjected to frequency sweep tests at $25^{\circ} \mathrm{C}$ over a frequency range of $0.1-10 \mathrm{~Hz}$, at a constant stress ( $1 \mathrm{~Pa})$. The elastic modulus $\left(\mathrm{G}^{\prime}\right)$ and loss modulus $\left(\mathrm{G}^{\prime}\right)$ were determined using the Rheology Modifier Advantage Control AR software.

\section{Syringeability Tests}

Syringeability tests were performed in a stress strain tester model DL-2000 (EMIC, São José do Pinhais, SP, Brazil) with a cell charge of $10 \mathrm{kgf}$ in the compression mode, using a glass syringe (1 $\mathrm{mL}$ ) with an internal diameter of $9.8 \mathrm{~mm}$ and a height of $97.8 \mathrm{~mm}$, equipped with a $21-\mathrm{G}$ needle $(25 \times 8 \mathrm{~mm})$, fixed in a specific device coupled to the tester. The FPS samples were carefully transferred to the interior of the syringe, avoiding the formation of air bubbles, to a fixed height of $30 \mathrm{~mm}$. The syringe plunger was then moved at a speed of $2.0 \mathrm{~mm} \cdot \mathrm{s}^{-1}$ (compression mode) over a distance of $30 \mathrm{~mm}^{38}$

The minimum force required to start the sample flow was obtained from the graph generated by the TESC version 3.01 software (EMIC) and the work expended in extrusion or the work of syringeability $\left(\mathrm{W}_{\mathrm{S}}, \mathrm{N} . \mathrm{mm}\right)$ was calculated from the area under a curve of force versus distance using the Origin ${ }^{\circledR} 6.1$ version software (OriginLab Corporation, Northampton, MA). The tests were performed at room temperature, and the results for each formulation were averaged from 5 separate runs.

\section{Hydration Studies}

Hydration studies were performed gravimetrically using a 15mm cylindrical plastic device fitted at one end with a mesh that had a porosity of $138 \times 75 \mu \mathrm{m}$, which contained the test sample within the device. Samples from each FPS (160 mg) were placed into the device, weighed (M1), and immersed in $10 \mathrm{~mL}$ of phosphate buffer ( $0.1 \mathrm{M}, \mathrm{pH} 7.0)$ at room temperature. At predetermined incubation times (i.e., $0.5,1,2,3,4,5,6$, and 24 h), each device was removed from the buffer, blotted dry and reweighed (M2).

The percentage increase in mass as a result of water uptake (Wu) was calculated using Equation 4. ${ }^{39}$ All experiments were performed at least in triplicate.

$W u(\% w / w)=\frac{(M 2-M 1)}{M 2} \times 100$

\section{In Vitro Assessment of Mucoadhesion}

Porcine cheek was obtained from a local slaughterhouse (Frigorífico Olhos D'agua, Ipuã, SP, Brazil) and was collected immediately after the animals were slaughtered and kept at $4^{\circ} \mathrm{C}$ during transport to the laboratory. Then, the cheek was rinsed with water, and the mucosa was separated from the muscular layer by cutting the loose connective fibers with a scalpel, preserving the basal membrane, and stored at $-80^{\circ} \mathrm{C}$ before use.

Immediately before the use, the mucosa was defrosted, cut into discs ( $1 \mathrm{~cm}$ inner diameter) using a stainless steel punch and incubated with artificial saliva ${ }^{40}$ for 5 min to moisten the tissue and hydrate the sample. The mucoadhesive properties were evaluated by a stress strain tester model DL-2000 with a cell charge of $10 \mathrm{kgf}$ in the tension mode.

Samples of the mucosa were fixed on acrylate plates using cyanoacrylate adhesive with the mucous layer upward. The plates were attached to the inferior and superior accessories of the equipment. This system was maintained at $37 \pm 1^{\circ} \mathrm{C}$ by a thermostatic bath. The FPS test sample $(0.1 \mathrm{~g})$ was smeared in a thin layer with a syringe onto the buccal mucosa that was attached to the inferior accessory.

Then, the superior instrument probe was lowered at speed rate of $0.5 \mathrm{~mm} / \mathrm{s}$ until contact with the test sample was made, and a constant compression of $0.5 \mathrm{~N}$ was maintained. After $3 \mathrm{~min}$ of contact, the superior probe was withdrawn at a rate of $0.5 \mathrm{~mm} / \mathrm{s}$. 
The peak detachment force (mucoadhesion strength, $S_{M}$ ) was calculated automatically with the TESC version 3.01 software. The mucoadhesion work $\left(\mathrm{W}_{\mathrm{M}}\right)$ corresponding to the area under a curve of force versus distance was calculated using the Origin ${ }^{\circledR} 6.1$ version software. The experiments were performed in quintuplicate.

\section{Results and discussion}

\section{PLM and SAXS Studies}

PLM is a technique that qualitatively identifies different phases by their texture. All FPSs were evaluated by PLM and showed a dark field, indicating isotropic behavior (Supplementary Fig. 1). These results suggest that the FPS was characterized as a micellar or cubic phase (both are isotropic and present a dark field). To confirm the phase indicated by PLM and evaluate the influence of the MO/CREM ratio, and the $W_{C}$ on the formation of the FPS, SAXS studies were performed. Figure 1 shows representative SAXS curves [scattering intensity versus scattering vector $(q)$ ] for some FPS and neat MO for comparison.

The SAXS pattern of neat MO shows 2 distinct scattering peaks that result from intermolecular correlations related to the electron density difference between the oxygen-rich portion (the polar portion of the glycerol moiety) and the nonpolar portion composed of hydrocarbon chains with a low electron density. ${ }^{42}$

The first peak is narrower and stronger in intensity and centered at a low $q$-value of approximately $1.30 \mathrm{~nm}^{-1}$ and suggests the formation of a structured phase. In fact, in the solid state, MO can present a lamellar phase due to structural organization of the hydrophilic "head group" (glyceryl group) and the hydrophobic "tail" (the hydrocarbon chain). ${ }^{23}$ The second broad scattering peak is weaker and centered at a $q$-value of approximately $2.00 \mathrm{~nm}^{-1}$ typical of dispersed system exhibiting $\mathrm{L}_{2}$ phase. ${ }^{41}$ These results suggest that neat MO consists of a lamellar plus $\mathrm{L}_{2}$ coexisting phases.

The SAXS curves for MC025W5 and MC025W10 (Supplementary Fig. 2a) have a profile similar to MC025W15 (Fig. 1) with a broad peak that has a $q$ value of approximately $0.8 \mathrm{~nm}^{-1}$, which is characteristic of the fluid isotropic phase $\left(\mathrm{L}_{2}\right){ }^{41}$ This behavior is typical of an aqueous micellar solution formed by a surfactant in water.

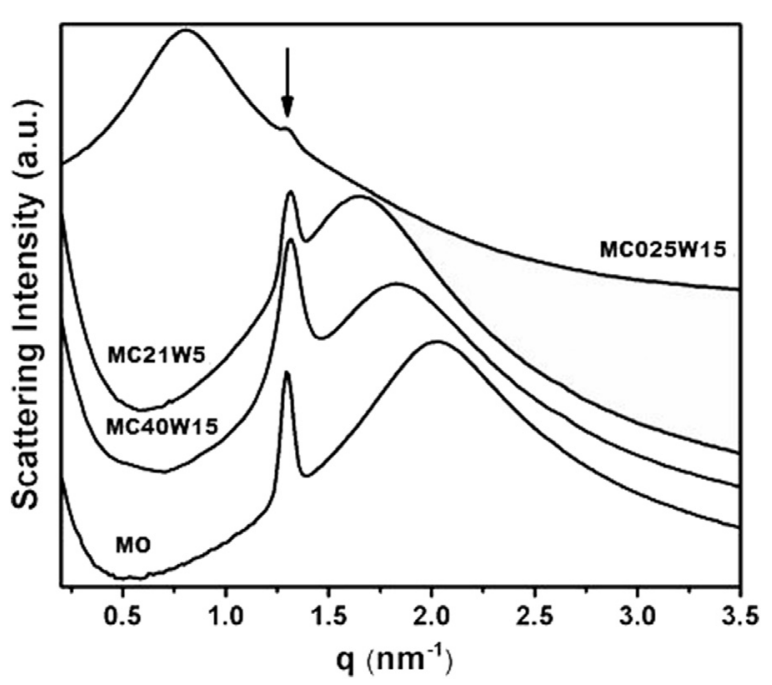

Figure 1. Representative SAXS patterns of liquid crystalline phase precursors MC025W15, MC21W5, MC40W15, and neat Myverol ${ }^{\circledR}$ 18-92 K (MO).
In this study, for a FPS with a MO/CREM ratio of 0.25 , CREM (the surfactant) is in excess compared with MO (at a 4-fold increase) and forms a micellar system in the presence of water that contributes to the SAXS curve profile and confirms the dark field observed by PLM. The water content did not significantly affect the structure because the profile of the SAXS curves did not vary with an increasing amount of water for a MO/CREM ratio of 0.25 .

An additional, but much smaller peak, is also visible at a q-value approximately $1.30 \mathrm{~nm}^{-1}$ (highlighted by an arrow), which can be attributed to presence of small quantities of lamellar phase from MO (Fig. 1).

An increase in the MO/CREM ratio from 0.25 to 2.1 induces structural changes in the FPS, as demonstrated by the SAXS curves for MC21W5 (the profile is the same for MC21W10 and MC21W15, Supplementary Fig. 2b). It is noteworthy that the increase in $\mathrm{MO} /$ CREM did not lead to a formation of a lamellar, cubic, or hexagonal LCP, but the profile was the same as observed for neat MO.

This behavior is even more evident when the ratio is increased to 4.0 (MC40W15) at which 2 broad peaks are still evident (Fig. 1). The first peak is fixed and insensitive to variations in the MO/CREM ratio, whereas the second broad peak is shifted from 1.65 to 1.83 $\mathrm{nm}^{-1}$ at a MO/CREM ratio of 4.0. These results indicate that the $\mathrm{W}_{\mathrm{C}}$ used in the formulations did not affect the FPS formation at any $\mathrm{MO} / \mathrm{CREM}$ ratio and did not form a characteristic LCP.

\section{Rheological Behavior}

\section{Steady Shear Rate Sweep Test}

Flow properties must be considered for a gel that is administered through a syringe, along with adequate storage and application at specific sites, for their possible effects on drug release. ${ }^{31}$ Figures 2a, 2b, and 2c show the rheological behavior for $\mathrm{MO} /$ CREM ratios of $0.25,2.1$, and 4.0 , respectively, at several $W_{C}$. Experiments were performed at $25^{\circ} \mathrm{C}$, simulating storage and syringe administration. ${ }^{43}$

For MC025W5, MC025W10, and MC025W15 (Fig. 2a), the shear stress behaved was a linear function of the shear rate, indicating Newtonian behavior that increased linearly with the $W_{C}$. Such behavior is typical of a micellar system, as confirmed by SAXS and PLM (Fig. 1). However, when the MO/CREM ratio was increased to 2.1 or 4.0 , the system was no longer Newtonian but viscoelastic instead (Figs. 2b and 2c). Rheograms showed a nonlinear relation between the shear stress and the shear rate, which was characteristic non-Newtonian behavior; that is, at a constant pressure and temperature, the viscosity is dependent on shear rate. ${ }^{44}$

MC21W5, MC21W10, and MC21W15 displayed viscoelastic behavior with a small yield stress value $\left(\sigma_{0}\right)$, which indicates that these systems have plastic behavior. According to Barnes, ${ }^{45}$ materials that display such behavior, known as soft solids, have the material capacity of being molded but require a minimal force, called the yield stress, to start flowing. This phenomenon has been attributed to the formation of a tridimensional network. ${ }^{31}$ These findings suggest that the system became reorganized and a phase transition from micellar to LCP occurred. However, the results do not confirm this, which means that this behavior is simply due to the high amount of MO in the formulation.

Increasing the $\mathrm{W}_{\mathrm{C}}$ also increases the viscosity and contributes to the plastic behavior of the FPS, and an extreme effect was evident at a MO/CREM ratio of 4.0 (Fig. 2c). The MC40W15, with $15 \%$ water, had a high viscosity and consequently a high $\sigma_{0}$ (i.e., a high minimum shear stress value was required to disrupt the internal structure so flow could begin).

Moreover, different thixotropic levels are observed between systems with MO/CREM ratios of 2.1 and 4.0, with more pronounced thixotropic levels at a MO/CREM ratio of 4.0. Thixotropic 

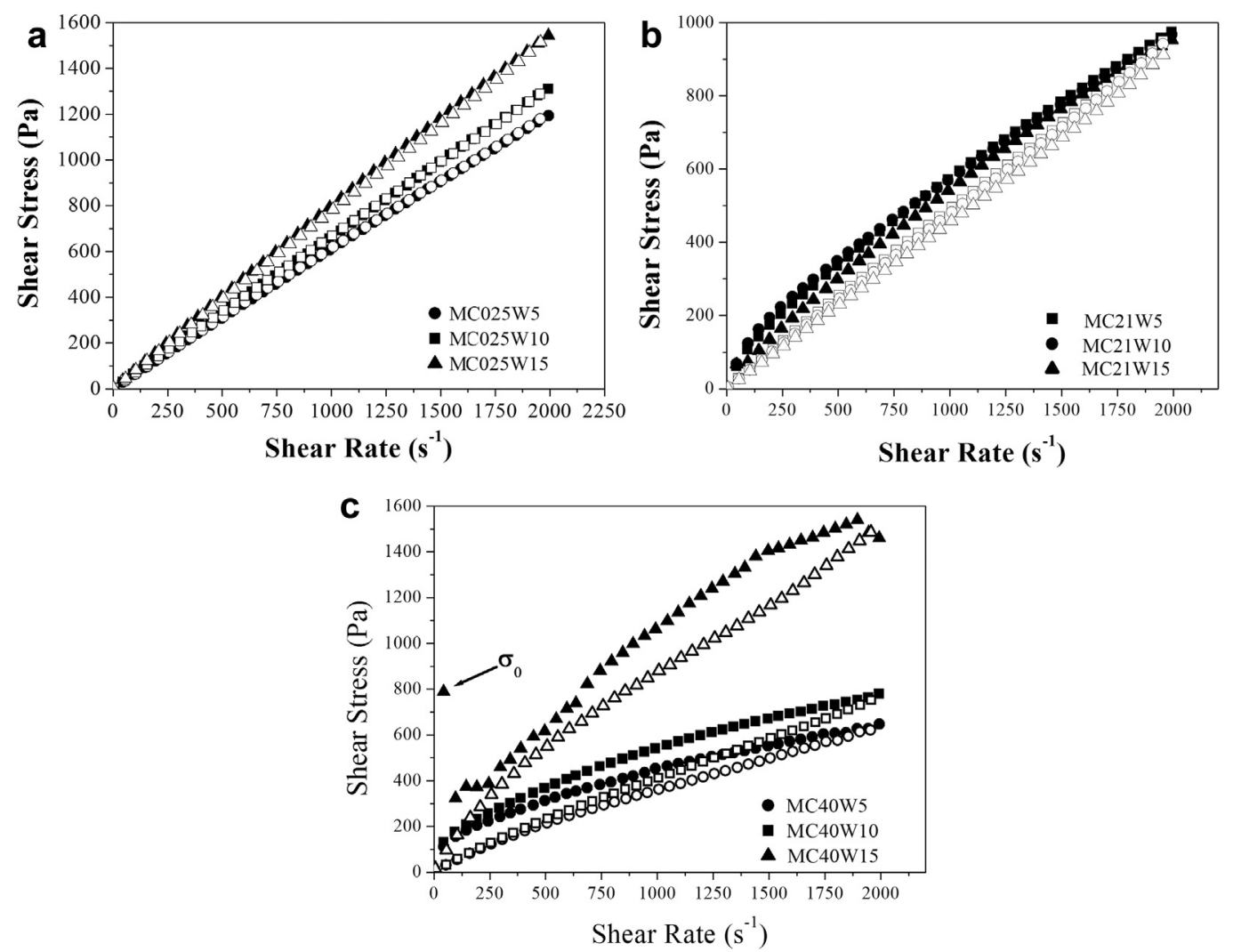

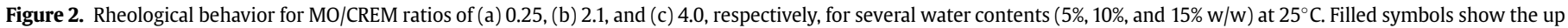
curves and open symbols the down curves. Relative standard deviation of at least 3 replicate tests was less than $5 \%$.

behavior is the ability to recover the original flow properties, irrespective of time. ${ }^{31,45}$ The thixotropic level for MC40W15 is higher than for the other samples. These results suggest that MO and water promote the formation of a thixotropic formulation.

To quantitatively determinate the change in the rheological properties of the FPS as a function of the MO/CREM ratio and the $W_{C}$, the data were calculated by the power law, that is, the Ostwaldde Waele model (Eq. 3). Most coefficients of determination $\left(\mathrm{R}^{2}\right)$ obtained were near 0.999, which indicated agreement between the model and the experimental data. The system MC40W15 was an exception; its viscosity, and $\sigma_{0}$ are high enough to disable the model fit. The Oswald-de Waele power law was used to quantify the shear-thinning behavior of the system: for a Newtonian system, $n=1$; for a system which exhibits shear-thinning behavior, $n<1$; and for a system which exhibits shear thickening, $n>1 .^{37}$

The $n$ values were close to 1 for all FPS with a MO/CREM ratio of 0.25 , which indicated Newtonian behavior, and the values of $k$ for MC025W5, MC025W10, and MC025W15 were on average 0.72, 0.69 , and 0.96 Pa.s, respectively, which confirmed the low viscosity.

MC21W5, MC21W10, and MC21W15 showed plastic flow behavior with moderate thixotropy (Fig. 2b). These 3 FPS had similar rheological behavior, with $k$ values between 1.63 and 2.28 Pa.s, which indicated that the viscosity increased with an increase in $\mathrm{MO}$ and the $\mathrm{W}_{\mathrm{C}}$. In this case, increasing the WC was correlated with a linear rate decrease of $k\left(\mathrm{R}^{2}=0.76\right)$.

The $n$ values of approximately 0.8 confirmed shear-thinning behavior, with minor thixotropy. MC40W5 and MC40W10 (Fig. 2c) had a higher thixotropy, shear-thinning (both 0.5) and viscosity (13.5 and 15.1 Pa.s, respectively) than the other systems. MC40W15 showed a high yield stress value $\left(\sigma_{0}\right)$ of approximately $800 \mathrm{~Pa}$, which contradicts the power law fit.
The gradual increase of the MO/CREM ratio and the $\mathrm{W}_{\mathrm{C}}$ in each FPS produced a slight decrease in the $n$ value (1.0 to 0.5 ). This confirmed that the increase in MO content had a greater effect on the plasticity of the system, with concomitant increases in the consistency index and the apparent sensitivity of viscosity to shear rate.

\section{Oscillatory Measurements}

Several research groups have used dynamic oscillatory rheological measurements in conjunction with PLM and SAXS to obtain complete information on in situ structural events in the transitions of FPS to LCP, in addition to the coexistence of transition phases. ${ }^{46,47}$ These techniques contributed to the understanding and characterization of the physicochemical properties of the FPS. The viscoelastic properties, such as the storage modulus $G^{\prime}$ and loss modulus $G^{\prime}$, were evaluated by sweeping the frequency.

An FPS with a MO/CREM ratio of 0.25 did not demonstrate viscoelastic properties, which indicated a low apparent viscosity and predominantly liquid behavior (Supplementary Fig. 3). At higher MO/CREM ratio such as 2.1 or 4.0 , the viscoelastic properties were greatly affected by the $\mathrm{W}_{\mathrm{C}}$. A better understanding can be gained from the $G^{\prime}$ and $G^{\prime \prime}$ moduli of the FPS (MC21W5, MC21W15, and MC40W15) that are presented in Figure 3.

An increase in the $W_{C}$ at a constant MO/CREM ratio (2.1), MC21W5 (5\% $\mathrm{W}_{\mathrm{C}}$ ) showed a dependence on the frequency and a "liquid-like" viscosity; $G^{\prime}<G^{\prime}$ ". The relation $G^{\prime}>G^{\prime}$ held for MC21W15 $\left(15 \% W_{C}\right)$ across the entire frequency range tested, indicating "gel-like" behavior; that is, that water favored the formation of the liquid crystalline phase. The same behavior was observed for FPS with a high MO/CREM ratio (4.0). 


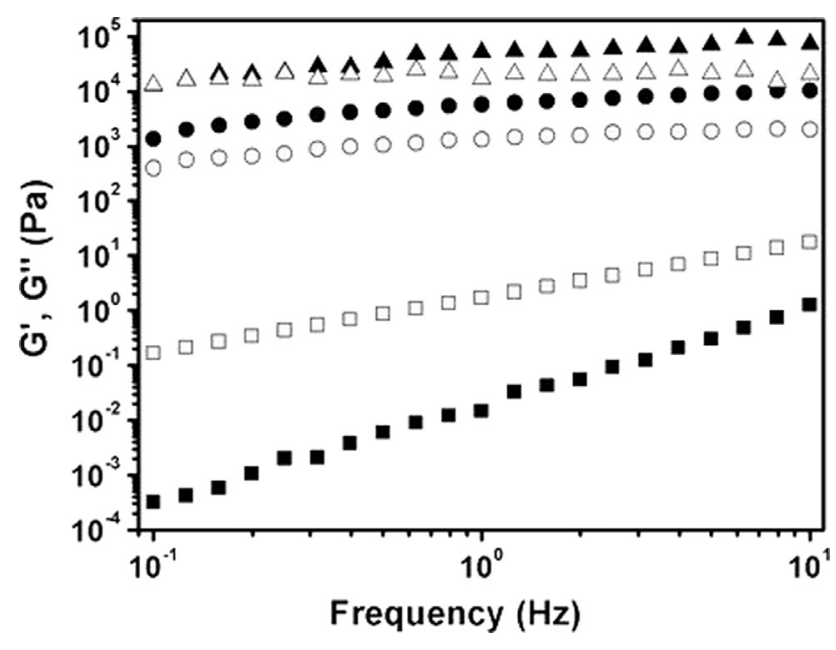

Figure 3. Oscillatory measurements showing the frequency dependence of the storage moduli G' (filled symbols) and loss moduli G" (open symbols) of MC21W5 ( $\mathbf{a}$ ), MC21W15 ( $)$, and MC40W15 ( $\boldsymbol{\Delta})$ at $25^{\circ} \mathrm{C}$.

However, for MC40W15, G' > G' and independent of the oscillatory frequency, indicating the formation of a strong gel network. According to Cordobés et al., ${ }^{48}$ the existence of this plateau region at a high frequency index is an important criterion for the identification of a gel-like network.

\section{Syringeability}

The syringeability work is an important parameter to evaluate during the development of a DDS to be sustained at a specific site, such as the vaginal or nasal cavity, the conjunctival sac, or the periodontal pocket. For a successful clinical administration, the delivery system must have suitable flowability for easy application into the desired mucosal sites. 7,38

The work was characterized by the extrusion of the system though a syringe fitted with a needle and increases with the $\mathrm{MO} /$ CREM ratio and the $\mathrm{W}_{\mathrm{C}}$ (Table 1 ), due to the increased viscosity and structure of the system, as shown by the rheological and SAXS measurements.

For a FPS with a MO/CREM ratios of 2.1 and 4.0, the increase in the $\mathrm{W}_{\mathrm{C}}$ correlated with a linear increase of the rate of the syringeability work $\left(\mathrm{R}^{2}=0.86\right.$ and 0.78 , respectively). These trends are closely related to the increase in the viscosity as a function of increasing $W_{C}$ in the FPS. MC40W15 had the highest value of the series (484.26 N.mm), which was expected on account of its plastic flow behavior (Fig. 2c) and the high yield stress value required to start the material flow. ${ }^{45}$ However, MC21W5 and MC21W10 had lower syringeability work values than the other FPS $(p<0.05)$, likely due to the pseudoplastic behavior coupled with moderate thixotropy, which are desirable properties for the development of a syringeable system.

Syringeability studies for intraperiodontal pocket delivery are still scarce in the literature. Some authors consider that work values between 10 and $380 \mathrm{~N} . \mathrm{mm}$ are adequate for easy extrusion. ${ }^{38}$ However, most reported assays of the syringeability of semisolid systems for application in the periodontal pocket were carried out using syringes without needles.

We decided to use syringes fitted with needles of a specific diameter because of the challenges of administering semisolid formulations in clinical dental procedures, primarily the limited space for insertion available in the periodontal pocket ( $4 \mathrm{~mm}$ up to $13 \mathrm{~mm}) .{ }^{49}$ The use of a needle can be justified by an accurate evaluation of the feasibility of DDS to be administered by this route.

\section{Water Uptake (Wu)}

The $\mathrm{Wu}$ studies are greatly relevant to the design of an LCP precursor as an injectable controlled DDS. Wu is particularly important in systems based on amphiphilic compounds such as MO; the rate of water absorption can affect the in situ transition (or self-assembly) of the FPS to the LCPs and consequently, the speed and duration of the drug-release and its stability after exposure to excess water. $^{32}$

The study the $\mathrm{Wu}$ may therefore provide data on the kinetics of drug release and the percentage of water that is required to initiate the transition from the precursor system to the liquid crystalline mesophase. During the buffer incubation, the FPS containing a high proportion of CREM (a MO/CREM ratio of 0.25 ) quickly absorbed water and completely disintegrated before $1 \mathrm{~h}$. In this case, the instability in the presence of excess water (disintegration effect) showed that the formation of a micellar system could be attributed to the partial replacement of MO by CREM. This effect could be due the hydrophilic characteristics of CREM, which is a surfactant with a high HLB (12 to 14), which promoted the complete solubilization of the MO under conditions of full hydration. Consequently, nonstructural transitions into the LCP did not occur, and the swelling profile of these micellar systems could not be obtained.

The rates of Wu by FPS at MO/CREM ratios of 2.1 and 4.0 are shown in Figure 4. Although the amounts of MO are different (Table 1 ), the rates of $\mathrm{Wu}$ within the first hour are statistically similar $(p>0.05)$. MC21W10, MC40W10, and MC40W15 displayed a slow increase in their rates up to $24 \mathrm{~h}$, with higher uptake percentages (Table 1 and Fig. 4), but the $\mathrm{MO} / \mathrm{H}_{2} \mathrm{O}$ ratios for these systems was almost the same, approximately 2 .

After $5 \mathrm{~h}$, most of the rates increased, but MC21W5 showed a different profile when compared the others in the group $(p<0.05)$. MC21W5 had a minimal $W u$ rate at a lower $W_{C}$ (Table 1 and Fig. 4), which could be due to the beginning of matrix erosion after $5 \mathrm{~h}$. This finding was further supported by the weight loss of the sample.

Most of the FPS had a rapid Wu during the first $6 \mathrm{~h}$, followed by an equilibrium uptake equivalent to $30 \%$ of the initial mass (w/w), that was independent of the initial $W_{C}$. These profiles are in agreement with Chang and Bodmeier, ${ }^{32}$ who used monolinoleate and monooleate (Myverol ${ }^{\circledR}$ 18-92 and Myverol ${ }^{\circledR} 18-99$, respectively). Taking into account the rapid hydration process, an LCP precursor would most likely be useful in applications in which the rapid initial release of a drug is desired, or for prolonged release from a poorly hydrated region, for example, from the periodontal pocket, ocular wound healing, and so forth.

Interestingly, after the rapid Wu during the first hours, the FPS with MO/CREM ratio 2.1 and 4.0 became slower because of the diffusion of water into the matrices on account of the structural

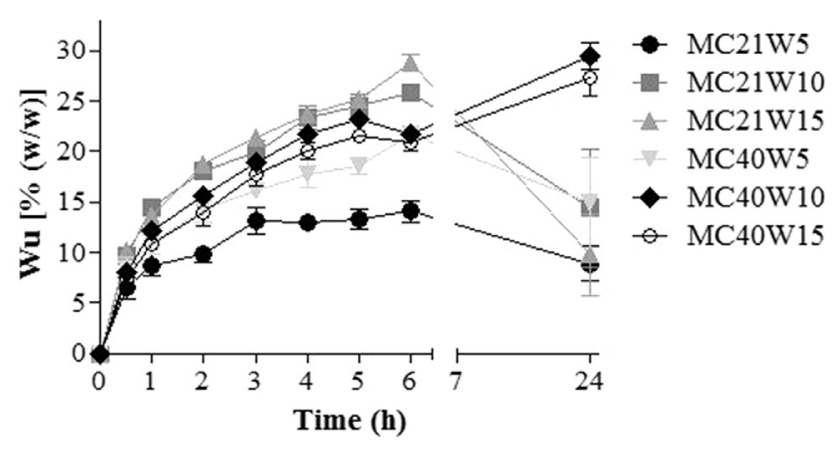

Figure 4. Water uptake profiles of liquid crystalline phase precursors incubated in buffer solution at $25^{\circ} \mathrm{C}$. Data represent mean \pm standard deviation, $n=3$. 
transition from the FPS to the viscous cubic phase, which is in accord with the SAXS pattern (Fig. 5).

As described elsewhere, ${ }^{20,50}$ most monoglyceride matrices have a diffusion-controlled release mechanism, in which the rate of drug release is controlled by the diffusion of the molecules through the LCP, which decreases with time because of the greater distance over which the drug must diffuse through the matrix to the exterior. This phenomenon occurs over time, retarding the drug release, providing the sustained release of a drug. Nevertheless, the initial $\mathrm{W}_{\mathrm{C}}$ and $\mathrm{Wu}$ rate are factors that could affect drug release.

\section{Self-Assembly Properties}

The study of self-assembly in excess of water was investigated by SAXS experiments. Peaks characteristic of the cubic phase ${ }^{46}$ can be observed in Figures $5 a$ and $5 b$. The LCP was determined from the relation between the peak distance and the scattering vector $(q)$ according to the Bragg equation. ${ }^{29}$ To calculate the correlation distance between the scattering objects, we used the equation $d=2 \pi / q_{\max }$, where $q_{\max }$ was the scattering vector of the first order peak.

The individual type of cubic phase is distinguished by a characteristic ratio; Pn3m: $\sqrt{2}, \sqrt{3}, \sqrt{4}, \sqrt{6} \ldots$, e $\operatorname{Im} 3 \mathrm{~m}: \sqrt{2}, \sqrt{4}, \sqrt{6} \ldots$ The diffraction patterns show at least 6 characteristic peaks for the diamond type Pn3m cubic phase. ${ }^{29,51}$ The phase is an ordered 3dimensional water-lipid system in which the lipids are organized in a highly curved, interwoven bilayer network. ${ }^{52}$

Different self-assembly structures, such as micelle systems (normal and reverse), microemulsions and LCP, may be formed after further hydration, assuming an appropriate ratio of surfactants and amphiphilic molecules, such as MO is present. ${ }^{22,25,53} \mathrm{~A}$ cubic phase was not formed at low ratios of MO/CREM (0.25), independently of the $\mathrm{W}_{\mathrm{C}}$. Increasing the hydrophobicity to average and high MO/CREM ratios (2.1 and 4.0, respectively) produced a FPS with a greater degree of structural order, on account of the coexistence of regions in transition to the LCP.

Figure 5a shows the SAXS patterns of MC21W5 and MC21W15 after $\mathrm{Wu}$. Despite the change of the $W_{C}$ (5\%-15\%), the LCP did not change. Both of these LCPs had a cubic Pn3m with the lattice parameters of cubic phase, $\mathrm{a}=7.84 \mathrm{~nm}$ and $7.76 \mathrm{~nm}$, respectively. The SAXS curves for MC40W5 and MC40W15 after water uptake are shown in Figure 5b. MC40W5 showed a phase mixture of Pm3n and Im $3 \mathrm{~m}$ as observed by Yaghmur et al. ${ }^{54}$ and MC40W5 showed only a Pm3n phase.

Parameter "a" changed from $7.6 \mathrm{~nm}$ to $5.9 \mathrm{~nm}$ when the $\mathrm{W}_{\mathrm{C}}$ was increased from $5 \%$ to $15 \%$. When the MO/CREM ratio was increased while $\mathrm{W}_{\mathrm{C}}$ was fixed at 5\% (MC21W5 and MC40W5), the smaller ratio produced a pure $\mathrm{Pn} 3 \mathrm{~m}$ cubic phase but the MO/CREM ratio of
4 produced a mixture. However, the lattice parameters did not show a relevant difference (7.8 $\mathrm{nm}$ and $7.6 \mathrm{~nm}$, respectively). Nonetheless, when $W_{C}$ was $15 \%$, increasing the MO/CREM ratio from 2.1 to 4.0 led to a significant reduction in the lattice parameter from $7.8 \mathrm{~nm}$ to $5.9 \mathrm{~nm}$.

A few studies have been performed on MO/CREM systems for drug delivery, and the principal factors responsible for the selfassembling effect were found to be the variation in the lipid composition, the $W_{C}$, and the physicochemical properties of the surfactants. ${ }^{51,54}$ Shah and Paradkar ${ }^{35}$ observed that hydrophilic additives (Hydrophilic-Lipophilic Balance, HLB between 7 and 11) to the MO matrices resulted in less self-assembly when compared with the same matrix with lipophilic additives (HLB between 1.5 and 5.0). It is hypothesized that hydrophilic domains may cause the reduced availability of water for the liquid crystalline mesophase and, consequently, irregularities in the LCP formation.

CREM is a hydrophilic surfactant with a HLB ranging from 12 to 14 , which may have contributed to the organization of the LCP precursor systems in this study. It also enabled the preparation of a more fluid system, compatible with administration by syringe, despite being previously structured for in situ transition to the LCP.

At a water excess $(40 \% \mathrm{w} / \mathrm{w})$, systems with MO/CREM ratios of 2.1 and 4 undergo a transition from an intermediate liquid crystal state to the cubic structure of the Pn $3 m$ phase. Furthermore, the results confirm that an increasing MO content had a key role in selfassembly by providing the transition to the mesophase at equilibrium with the excess water. The higher content of CREM on the FPS with MO/CREM ratio of 0.25 was unstable in the presence of excess water, and disintegrated. This observation led us to use a higher CREM content than Yaghmur et al. ${ }^{54}$

\section{Mucoadhesive Properties}

The main advantage of mucoadhesive DDS is the increased residence time in the administration site. During slow, continuous drug release, they can reduce the administration frequency and often increase both patient compliance and treatment efficacy. For buccal purposes, this type of DDS must be engineered to surmount several challenges, for example, pathological conditions in the administration region; shearing caused by speech, buccal hygiene, and chewing; and continuous salivary flow. ${ }^{13}$ This latter is the main factor responsible for the lack of the success of hydrophilic polymers. Thus, research interest has been shifting toward the implementation of materials that are biodegradable and nonaqueous or stable in an aqueous medium, especially those that self-assemble and are mucoadhesive in such a medium, such as glyceryl monolinoleate. ${ }^{20,55}$
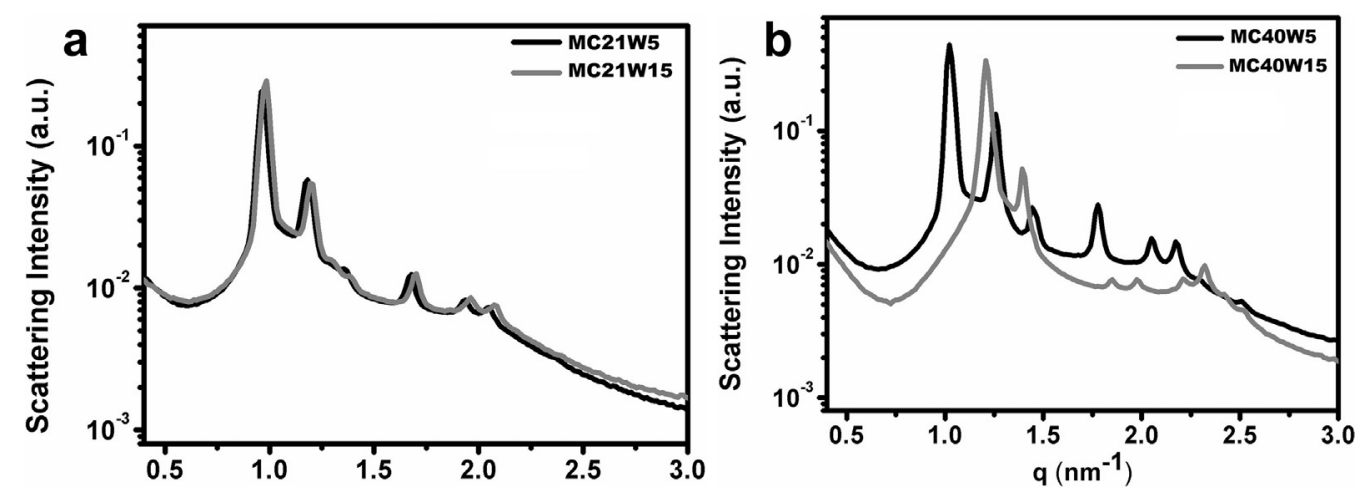

Figure 5. Representative SAXS pattern of liquid crystalline phases (a) MC21W5 and MC21W15 and (b) MC40W5 and MC40W15 after water uptake at $25^{\circ} \mathrm{C}$. 
Porcine buccal mucosa from the cheek has been widely used as a tissue model for in vitro mucoadhesion studies because its biochemical and histological properties approximate those of human tissue. ${ }^{13}$ In this study, the mucoadhesive properties of the FPS were evaluated using porcine cheek mucosa because their primary use was envisioned as buccal drug delivery platforms, especially in the periodontal pocket. Table 1 summarizes the results of the mucoadhesion strength $\left(\mathrm{S}_{\mathrm{M}}\right)$ and mucoadhesion work $\left(\mathrm{W}_{\mathrm{M}}\right)$.

The $S_{M}$ and $W_{M}$ values varied from $0.09 \pm 0.01 \mathrm{~N}$ to $0.19 \pm 0.04 \mathrm{~N}$ and from $0.36 \pm 0.04 \mathrm{~N} . \mathrm{mm}$ to $1.17 \pm 0.17 \mathrm{~N} . \mathrm{mm}$, respectively. The $\mathrm{S}_{\mathrm{M}}$ results are in agreement with Geraghty et al., ${ }^{56}$ who reported detachment force values ranging from 0.06 to $0.18 \mathrm{~N}$ with glyceryl monolinoleate. In addition, compared with the mucoadhesive polymeric systems described elsewhere, ${ }^{57}$ although low, these values might be suitable for buccal purposes.

The $S_{M}$ of M025W5, M025W10, and M025W15 were significantly lower $(p<0.01)$ compared with the other FPS. This may be due to the low viscosity and $\mathrm{Wu}$ of these formulations during the transition into the LCP during the contact time with artificial saliva. Conversely, the FPS that had average and high MO/CREM ratios had greater average $S_{M}$ and $W_{M}$ values in excess artificial saliva $(p<0.05)$, which indicates the transition of the micellar structure toward the cubic crystalline phase in these systems, as evidenced by the PLM and SAXS analysis after Wu (Fig. 4). The phase transition enhanced the elastic properties of the system, which behaved like a gel, with a positive effect on the mucoadhesive properties. 7,31

These findings suggest 2 mechanism of mucoadhesion for these FPS: (1) mucoadhesion occurs on account of weak and nonspecific interactions between the formulation and the mucus, for example, hydrogen bonds, Van der Waals forces, and hydrophobic bounds (the adsorption theory of mucoadhesion), ${ }^{13}$ facilitated by the amphiphilic molecular structure of glyceryl monolinoleate; (2) the movement of water from the mucosa to the formulation generates a capillary flow that facilitates the attachment of both surfaces.

For a semisolid DDS, rupture or failure of the mucoadhesive bonds may occur at 3 different regions: (1) the hydrated layer of the mucoadhesive system; (2) the interface between the mucosa and the mucoadhesive system; and (3) at the mucus layer. In mucoadhesive systems with a high $S_{M}$, the failure occurs at the mucus layer, but for low and very low $S_{M}$, it occurs at the interface and the hydrated layer, respectively. ${ }^{13}$ Accordingly, the rupture for these FPSs could have occurred at the interface between the mucosa and the formulation.

\section{FPS Performance Interactions}

The significance of the complex results of the individual FPS characterizations (Table 1 ) require further investigation, as do the interactions of the performance indicators (dependent variables) and the factors studied (independent variables). To accurately determinate the interactions of MO/CREM ratio and $\mathrm{W}_{C}$ with the performance indicators, an ANOVA and correlation analyses were performed by the RSM. Tables with the complete ANOVA for each FPS property are not shown, but a summary of the RSM results is summarized in Table 2.

The response surface analysis allows the fitting of a polynomial equation of the dependent variables as a function of the significant factors to predict performance indicators. In Table 2, the bold values are the significant model coefficients, and their levels of significance are displayed as percentages. The sign indicates whether the effect is positive or negative. The high coefficients of determination of the fitted models are significant and indicate that these equations can be used to describe the experimental results.
Table 2

Summary of Response Surface Methodology (RSM) Results

\begin{tabular}{llllll}
\hline Coefficient & $k($ Pa.s $)$ & $n(-)$ & $\mathrm{W}_{\mathrm{S}}(\mathrm{N} \cdot \mathrm{mm})$ & $\mathrm{S}_{\mathrm{M}}(\mathrm{N})$ & $\mathrm{W}_{\mathrm{M}}(\mathrm{N} . \mathrm{mm})$ \\
\hline Intercept & -25.29 & $\mathbf{0 . 8 1}^{* * *}$ & $\mathbf{9 9 . 8}^{*}$ & $\mathbf{0 . 1 7}^{* * *}$ & $\mathbf{0 . 9 4}^{* * *}$ \\
$X_{1}$ & 92.79 & $-\mathbf{0 . 3 2}^{* *}$ & $\mathbf{5 5 . 5}^{*}$ & $\mathbf{0 . 0 3}^{*}$ & $\mathbf{0 . 2 2}^{*}$ \\
$X_{1}^{2}$ & 73.70 & -0.10 & $\mathbf{1 0 3 . 1}^{*}$ & -0.03 & -0.19 \\
$X_{2}$ & 86.10 & -0.07 & $\mathbf{6 4 . 9}^{*}$ & -0.01 & 0.02 \\
$X_{2}^{2}$ & 67.74 & -0.06 & 42.5 & -0.02 & 0.01 \\
$X_{1} \cdot X_{2}$ & 129.19 & -0.13 & 66.6 & -0.003 & 0.01 \\
$\mathrm{R}^{2}$ & 0.78 & 0.91 & 0.88 & 0.73 & 0.77 \\
\hline
\end{tabular}

$X_{1}$. MO/CREM, Myverol ${ }^{\circledR} 18-92 \mathrm{~K} /$ Cremophor $^{\circledR}$ EL ratio $(\mathrm{w} / \mathrm{w}) ; X_{2} \cdot \mathrm{W}_{\mathrm{C}}[\%(\mathrm{w} / \mathrm{w})]$, water content; $k$, consistency index; $n$, flow behavior index; $\mathrm{W}_{\mathrm{S}}$, work of syringeability; $\mathrm{S}_{\mathrm{M}}$, mucoadhesion strength; $W_{M}$, mucoadhesion work; $R^{2}$, coefficient of determination of regression model.

Bold values represent the model coefficient significant at ${ }^{*} 5 \%,{ }^{* *} 1 \%$, and ${ }^{* * *} 0.1 \%$.

The response surfaces of the performance indicators are shown in Figures $6 a-6 d$ as functions of the significant factors.

None of the factors studied significantly affected the consistence index $(k)$. However, the MO/CREM ratio had a linear negative effect on the flow behavior index ( $n$ ), that is, an increasing MO/CREM ratio decreased the $n$ (Fig. 6a). On the other hand, the MO/CREM ratio positively affected both the $S_{M}$ and $W_{M}$ as shown in Figures $6 c$ and $6 \mathrm{~d}$, respectively.

Figure $6 \mathrm{~b}$ shows the surface response of $\mathrm{W}_{\mathrm{S}}$ as a function of $\mathrm{MO} /$ CREM and $W_{C}$. The surface shows that MO/CREM had a positive nonlinear effect on the $\mathrm{W}_{\mathrm{S}}$. The nonlinear effect of MO/CREM was confirmed by the ANOVA (Table. 2), which had a significance level of $5 \%$ for the squared term $\left(\mathrm{MO} / \mathrm{CREM}^{2}\right)$.

To better understanding how the MO/CREM ratio, $\mathrm{W}_{\mathrm{C}}$ and the rheological behavior, syringeability, and mucoadhesion results are correlated, a correlation matrix was prepared (Supplementary Table 1). None of the performance indicators was significantly correlated with the $\mathrm{W}_{\mathrm{C}}$. Notwithstanding, the MO/CREM ratio was negatively correlated with $n$ and positively correlated with the $W_{M}$ parameters. As expected, the parameters relating to the rheological properties, that is, $k$ and $n$, were negatively correlated with each other. Conversely, the $\mathrm{W}_{\mathrm{S}}$ was positively correlated with $k$ and negatively correlated with $n$.

Finally, the parameters relating to the mucoadhesive properties $\left(\mathrm{S}_{\mathrm{M}}\right.$ and $\left.\mathrm{W}_{\mathrm{M}}\right)$ were positively correlated, which means that increasing one parameter increased the other, and vice versa. Overall, these results confirmed the trends discussed earlier and demonstrate that increasing the monoglyceride amount in the LPS increases the viscosity and consequently, increases the syringeability work and enhances mucoadhesion.

\section{Conclusions}

For the first time, the effects of Myverol ${ }^{\circledR} 18-92 \mathrm{~K}$, Cremophor ${ }^{\circledR}$ EL, and water content on the performance of an in situ gelling, liquid crystalline system have been systematically evaluated with a factorial design. The self-assembly profile, rheological behavior, syringeability, water uptake, and in vitro mucoadhesion were greatly affected by the relative contribution of the MO in FPS. The best performance was achieved with an average and high $\mathrm{MO}$ / CREM ratio, which had shear-thinning behavior with moderate thixotropy, viscoelastic properties and desirable syringeability and mucoadhesion. Moreover, these ratios may enable sustained drug delivery caused by the transition into the cubic phase after water is taken up.

Data arising from this report would be extremely useful for effective DDS engineering and its end usage properties. Further investigations will be performed to evaluate the in vitro drug delivery kinetics for the most promising LCP precursors. For that, both 
a

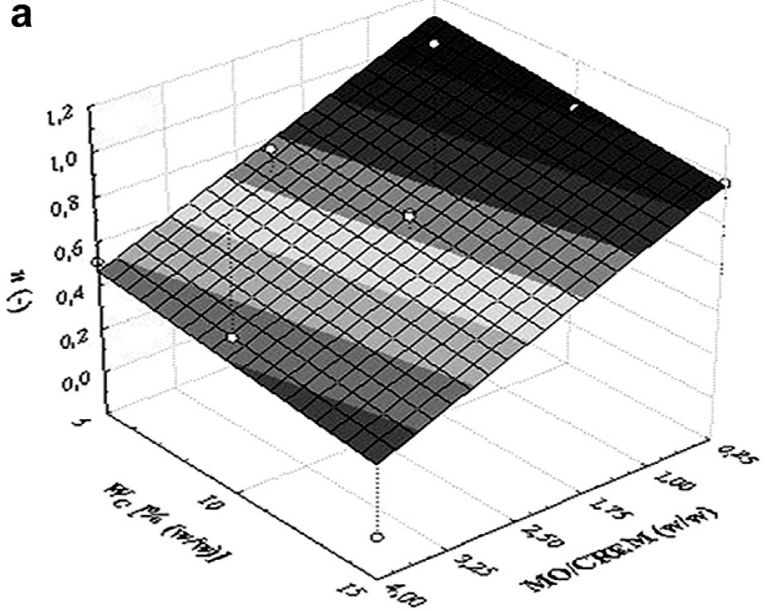

C

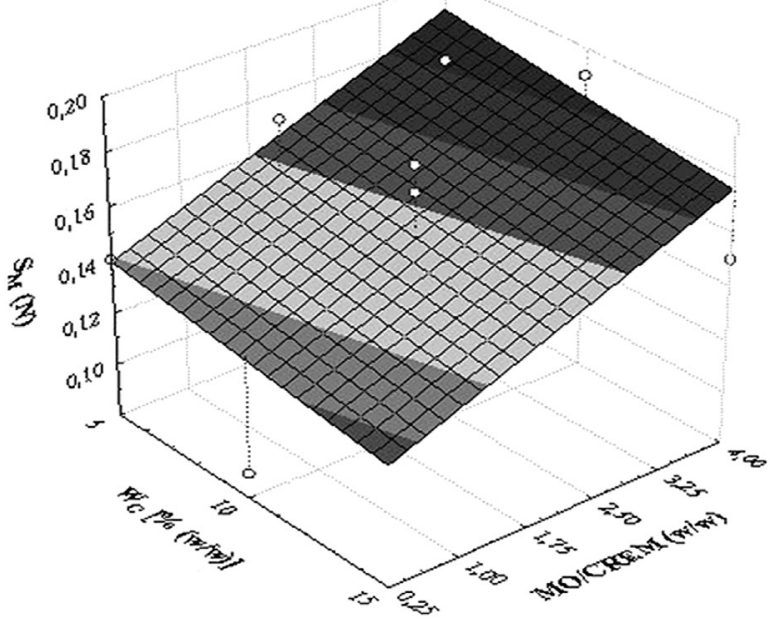

b

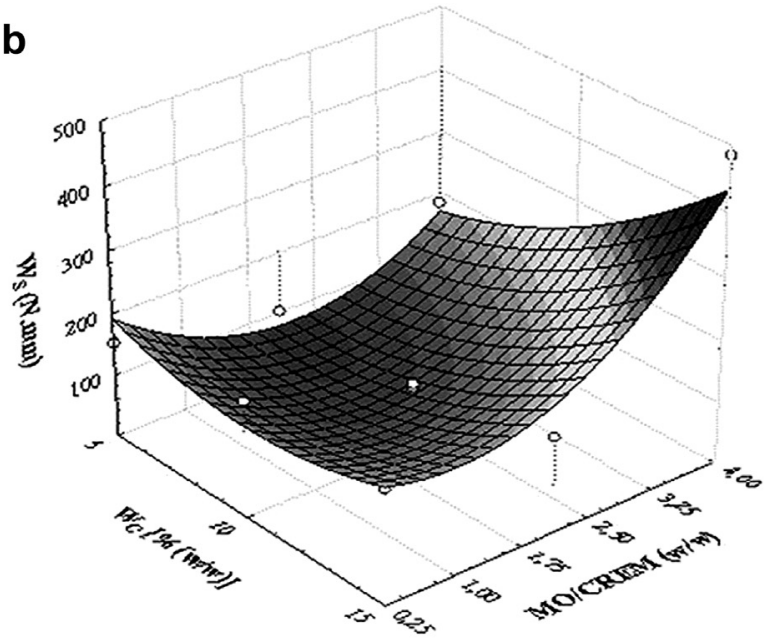

d

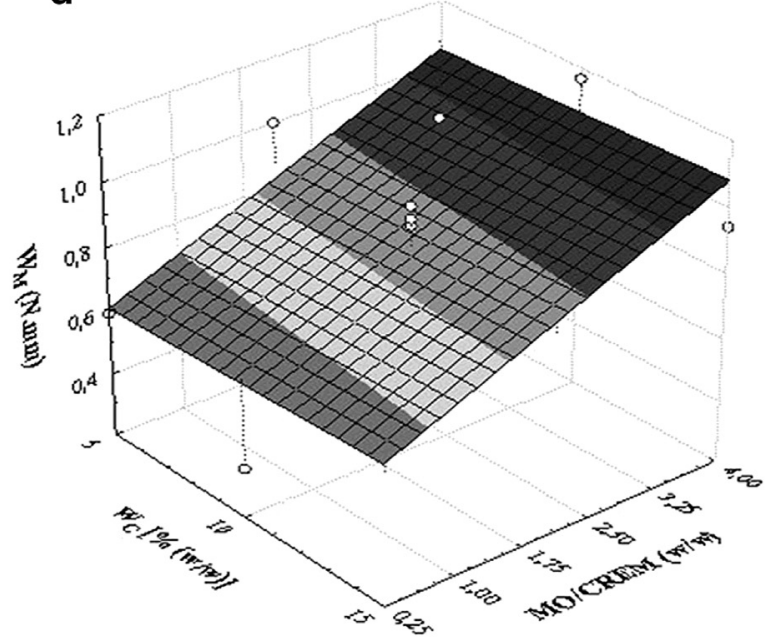

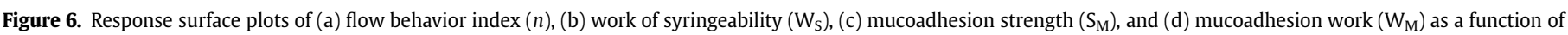
Myverol ${ }^{\circledR} 18-92 \mathrm{~K} /$ Cremophor $^{\circledR}$ EL ratio (MO/CREM) and water content $\left(\mathrm{W}_{\mathrm{C}}\right)$.

hydrophilic and lipophilic substances such as lidocaine hydrochloride and clindamycin, respectively, may be used as model drugs.

\section{Acknowledgments}

The authors greatly thank the LNLS (Project award number 12462) for SAXS measurements and Coordination of Improvement of Higher Education Personnel (CAPES) for financial support.

\section{References}

1. Carvalho FC, Campos ML, Peccinini RG. Nasal administration of liquid crystal precursor mucoadhesive vehicle as an alternative antiretroviral therapy. Eur J Pharm Biopharm. 2013;84:219-227.

2. Gratieri T, Gelfuso GM, Freitas O, Rocha EM, Lopez RFV. Enhancing and sustaining the topical ocular delivery of fluconazole using chitosan solution and poloxamer/chitosan in situ forming gel. Eur J Pharm Biopharm. 2011;79:320327.

3. Jones DS, Luciano M, Freitas O, et al. Rheological, mechanical and mucoadhesive properties of thermoresponsive, bioadhesive binary mixtures composed of poloxamer 407 and carbopol 974P designed as platforms for implantable drug delivery systems for use in the oral cavity. Int J Pharm. 2009;372:49-58.

4. Hou Q Chau DYS, Pratoomsoot C, et al. In situ gelling hydrogels incorporating microparticles as drug delivery carriers for regenerative medicine. J Pharm Sci. 2008;97:3972-3980.
5. Boyd BJ, Whittaker DV, Khoo S, Davey G. Lyotropic liquid crystalline phases formed from glycerate surfactants as sustained release drug delivery systems. Int J Pharm. 2006;309:218-226.

6. Esposito E, Carotta V, Scabbia A, et al. Comparative analysis of tetracyclinecontaining dental gels: poloxamer- and monoglyceride-based formulations. Int J Pharm. 1996;142:9-23.

7. Andrews GP, Donnelly L, Jones DS, et al. Characterization of the rheological, mucoadhesive, and drug release properties of highly structured gel platforms for intravaginal drug delivery. Biomacromolecules. 2009;10:2427-2435.

8. Araújo RR, Bruschi ML. Vaginal mucoadhesive drug delivery systems. Drug Dev Ind Pharm. 2012;38:643-652.

9. Cho HJ, Prabagar B, Eun-Kyoung P, et al. Poloxamer/cyclodextrin/chitosanbased thermoreversible gel for intranasal delivery of fexofenadine hydrochloride. J Pharm Sci. 2011;100:681-691.

10. Bruschi M, Jones D. Semisolid systems containing propolis for the treatment of periodontal disease: in vitro release kinetics, syringeability, rheological, textural, and mucoadhesive properties. J Pharm Sci. 2007;96:2074-2089.

11. Miani PK, Nascimento C, Sato S, Filho AV, Fonseca MJV, Pedrazzi V. In vivo evaluation of a metronidazole-containing gel for the adjuvant treatment of chronic periodontitis: preliminary results. Eur J Clin Microbiol Infect Dis. 2012;31:1611-1618.

12. Nair SC, Anoop KR. Intraperiodontal pocket: an ideal route for local antimicrobial drug delivery. J Adv Pharm Technol Res. 2012;3:9-15.

13. Carvalho F, Bruschi M, Evangelista R, Gremião M. Mucoadhesive drug delivery systems. Braz J Pharm Sci. 2010;46:1-17.

14. Khutoryanskiy V. Advances in mucoadhesion and mucoadhesive polymers Macromol Biosci. 2011;11:748-764.

15. Li H, Hardy RJ, Gu X. Effect of drug solubility on polymer hydration and drug dissolution from polyethylene oxide (PEO) matrix tablets. AAPS PharmSci Tech. 2008;9:437-443. 
16. Yu L, Li K, Liu X, et al. In vitro and in vivo evaluation of a once-weekly formulation of an antidiabetic peptide drug exenatide in an injectable thermogel. J Pharm Sci. 2013;102:4140-4149.

17. Zembko I, Ahmed I, Farooq A, et al. Development of disulfiram-loaded poly(lactic-co-glycolic acid) wafers for the localised treatment of glioblastoma multiforme: a comparison of manufacturing techniques. J Pharm Sci. 2015;104:1076-1086.

18. Ahmed T, Ibrahim H, Ibrahim F, et al. Development of biodegradable in situ implant and microparticle injectable formulations for sustained delivery of haloperidol. J Pharm Sci. 2012;101:3753-3762.

19. Couto RO, Sommerfeld SD, Dube K, De FO, Kohn J. Preliminarily development of a moisture-activated bioresorbable polymeric platform for drug delivery. Quim Nova. 2015;38:902-909.

20. Souza C, Watanabe E, Borgheti-Cardoso LN, De Fantini MCA, Lara MG. Mucoadhesive system formed by liquid crystals for buccal administration of poly(hexamethylene biguanide) hydrochloride. J Pharm Sci. 2014;103:39143923.

21. Campo L, Yaghmur A, Sagalowicz L, Leser ME, Watzke H, Glatter O. Reversible phase transitions in emulsified nanostructured lipid systems. Langmuir. 2004;20:5254-5261.

22. Kulkarni CV. Lipid crystallization: from self-assembly to hierarchical and biological ordering. Nanoscale. 2012;4:5779-5791.

23. Kulkarni CV, Wachter W, Iglesias-Salto G, Engelskirchen S, Ahualli S. Monoolein: a magic lipid? Phys Chem Chem Phys. 2011;13:3004-3021.

24. Fehér A, Urbán E, Eros I, Szabó-Révész P, Csányi E. Lyotropic liquid crystal preconcentrates for the treatment of periodontal disease. Int J Pharm. 2008;358:23-26.

25. Borgheti-Cardoso LN, Depieri LV, Diniz $\mathrm{H}$, et al. Self-assembling gelling formulation based on a crystalline-phase liquid as a non-viral vector for siRNA delivery. Eur J Pharm Sci. 2014;58:72-82.

26. Achouri D, Sergent M, Tonetto A, Piccerelle P, Andrieu V, Hornebecq V. Selfassembled liquid crystalline nanoparticles as an ophthalmic drug delivery system. Part II: optimization of formulation variables using experimental design. Drug Dev Ind Pharm. 2014;41:493-501.

27. Yaghmur A, Larsen SW, Schmitt M, et al. In situ characterization of lipidic bupivacaine-loaded formulations. Soft Matter. 2011;7:8291-8295.

28. Ren X, Svirskis D, Alany RG, Zargar-shoshtari S, Wu Z. In-situ phase transition from microemulsion to liquid crystal with the potential of prolonged parenteral drug delivery. Int J Pharm. 2012;431:130-137.

29. Seddon JM, Templer RH. Polymorphism of lipid-water systems. In: Sackmann L, Sackmann E, eds. Handbook of Biological Physics. Volume 1. London: Elsevier North Holland; 1995:97-160.

30. Tang TD, Brooks NJ, Jeworrek C, et al. Hydrostatic pressure effects on the lamellar to gyroid cubic phase transition of monolinolein at limited hydration. Langmuir. 2012;28:13018-13024.

31. Lee $\mathrm{CH}$, Moturi $\mathrm{V}$, Lee Y. Thixotropic property in pharmaceutical formulations. J Control Release. 2009;136:88-98.

32. Chang C-M, Bodmeier R. Effect of dissolution media and additives on the drug release from cubic phase delivery systems. J Control Release. 1997;46:215-222.

33. Chang CM, Bodmeier R. Binding of drugs to monoglyceride-based drug delivery systems. Int J Pharm. 1997;147:135-142.

34. Rangel-yagui CO, Pessoa-jr A, Tavares LC. Micellar solubilization of drugs. J Pharm Pharm Sci. 2005;8:147-163.

35. Shah MH, Paradkar A. Effect of HLB of additives on the properties and drug release from the glyceryl monooleate matrices. Eur J Pharm Biopharm. 2007;67: 166-174.

36. Kalepu S, Manthina M, Padavala V. Oral lipid-based drug delivery systems-an overview. Acta Pharm Sin B. 2013;3:361-372.
37. Barnes HA. A handbook of elementary rheology. South Glam: Aberystwyth: Intitute of non-Newtonian Fluid Mechanics, University of Wales; 2000.

38. Kelly HM, Deasy PB, Ziaka E, Claffey N. Formulation and preliminary in vivo dog studies of a novel drug delivery system for the treatment of periodontitis. Int J Pharm. 2004;274:167-183.

39. Perioli L, Ambrogi V, Rubini D, et al. Novel mucoadhesive buccal formulation containing metronidazole for the treatment of periodontal disease. $J$ Control Release. 2004;95:521-533.

40. Mashru RC, Sutariya VB, Sankalia MG, Parikh PP. Development and evaluation of fast-dissolving film of salbutamol sulphate. Drug Dev Ind Pharm. 2005;31:2534.

41. Polizelli MA, Telis VRN, Amaral LQ, Feitosa E. Formation and characterization of soy bean oil/surfactant/water microemulsions. Colloids Surf A. 2006;281:230236.

42. Li Y, Fabiano-tixier AS, Ruiz K, et al. Comprehension of direct extraction of hydrophilic antioxidants using vegetable oils by polar paradox theory and small angle X-ray scattering analysis. Food Chem. 2015;173:873-880.

43. Perioli L, Pagano C, Mazzitelli S, Rossi C, Nastruzzi C. Rheological and functional characterization of new antiinflammatory delivery systems designed for buccal administration. Int J Pharm. 2008;356:19-28.

44. Andrews GP, Jones DS. Rheological characterization of bioadhesive binary polymeric systems designed as platforms for drug delivery implants. Biomacromolecules. 2006; 7:899-906.

45. Barnes HA. Thixotropy-a review. J Nonnewton Fluid Mech. 1997;70:1-33.

46. Mezzenga R, Meyer C, Servais C, Romoscanu AI, Sagalowicz L, Hayward RC. Shear rheology of lyotropic liquid crystals: a case study. Langmuir. 2005;21: 3322-3333.

47. Libster D, Aserin A, Wachtel E, Shoham G, Garti N. An H II liquid crystal-based delivery system for cyclosporin A: physical characterization. J Colloid Interface Sci. 2007;308:514-524.

48. Cordobés BF, Franco JM, Gallegos C. Rheology of the lamellar liquid-crystalline phase in polyethoxylated alcohol/water/heptane systems. Grasas Y Aceites. 2005;56:96-105.

49. Medlicott N, Rathbone M, Tucker I, Holborow D. Delivery systems for the administration of drugs to the periodontal pocket. Adv Drug Deliv Rev. 1994;13: 181-203.

50. Lara MG, Bentley MVLB, Collett JH. In vitro drug release mechanism and drug loading studies of cubic phase gels. Int J Pharm. 2005;293:241-250.

51. Yaghmur A, Rappolt M, Østergaard J, Larsen C, Larsen SW. Characterization of bupivacaine-loaded formulations based on liquid crystalline phases and microemulsions: the effect of lipid composition. Langmuir. 2012;28:2881-2889.

52. Hruijff B. Lipids beyond the bilayer. Nature. 1997;386:129-130.

53. Jin L, Wang H, Yang Y. Thermodynamically controllable transition from 3D to 2D self-assembly of a hydrogelator induced by the phase behavior of triblock copolymers. J Colloid Interface Sci. 2013;393:53-57.

54. Yaghmur A, Campo L, Sagalowicz L, Leser ME, Glatter O. Control of the internal structure of MLO-based isasomes by the addition of diglycerol monooleate and soybean phosphatidylcholine. Langmuir. 2006;22:9919-9927.

55. Nielsen LS, Schubert L, Hansen J. Bioadhesive drug delivery systems I. Characterisation of mucoadhesive properties of systems based on glyceryl monooleate and glyceryl monolinoleate. Eur J Pharm Sci. 1998;6:231-239.

56. Geraghty P, Attwood D, Collett J, Sharma H, Dandiker Y. An investigation of the parameters influencing the bioadhesive properties of Myverol 18-99/water gels. Biomaterials. 1997; 16:63-67.

57. Cubayachi C, Couto RO, Gaitani CM, Pedrazzi V, Freitas O, Lopez RFV. Needlefree buccal anesthesia using iontophoresis and amino amide salts combined in a mucoadhesive formulation. Colloids Surf B Biointerfaces. 2015;136:11931201. 